\title{
UN DES ROLES DES CHAINES D'ABEILLES : LA TORSION DES RAYONS POUR LES RENDRE PARALLÈLES ENTRE EUX
} PAR

\author{
R. DARCHEN
}

Station de recherches apicoles, Bures-sur-Yvette.

Nous avons précédemment étudié les facteurs régissant le parallélisme des rayons et décrit la technique des Abeilles qui l'établissent. Ces insectes utilisent en particulier deux méthodes pour rendre parallèle un rayon placé anormalement :

I ${ }^{0}$ Ils allongent le rayon, vers le bas et sur les côtés, par des cellules dont le plan de base se rapproche de plus en plus du plan de base ordinaire, parallèle aux rayons voisins.

$2^{\circ}$ Ils entreprennent des travaux de démolition et de reconstruction sur la base et sur les parois des cellules du rayon pour les remettre en ordre.

A la fin de 1'œuvre, un observateur inexpérimenté aurait beaucoup de mal à retrouver les transformations effectuées.

Quelles régulations observe-t-on lorsqu'on présente aux Abeilles non plus un petit rayon, mais un fragment de cire gaufrée, en position perpendiculaire aux autres rayons? (fig. I).

( $\mathrm{I}_{\text {a }}$ cire gaufrée porte sur ses deux faces des empreintes mécaniques le la taille du fond des cellules).

\section{MATÉRIEL ET MÉTHODE DE TRAVAIL}

Au sommet d'une ruche Dadant, sur une barrette de bois entre deux cadres, est fixé un morceau de cire gaufrée de 3,5 cellules de large sur 20 de long (qui correspond à $20 \times 98 \mathrm{~mm}$ ou bien un autre de $24 \times$ I $40 \mathrm{~mm}$. Chaque morceau de cire est situé dans un plan vertical, orthogonal à la direction des plans des cadres de la ruche (fig. I). 
Dans les expériences préliminaires et la plupart des suivantes, la distance entre l'axe longitudinal de la barrette et celui des cadres voisins est à peu près celle qui est adoptée pour les cadres de la ruche, $37 \mathrm{~mm}$. Mais il est facile d'augmenter ou diminuer cette distance. Enfin, on peut aussi agir sur les faces des rayons voisins de la cire gaufrée en les recottvrant de divers écrans: plaque de métal, grillage.

Il faut tenir compte en ontre de la densité de la population d'Abeilles car bien souvent des résultats négatifs (absence de torsion) ne sont que la conséquence d'une quantité d'Abeilles insuffisante pour réchauffer ou pour tordre la cire. Dans ces conditions, les insectes se contentent de souder les bords des bandes de cire sur les cellules ou les écrans des rayons voisins.

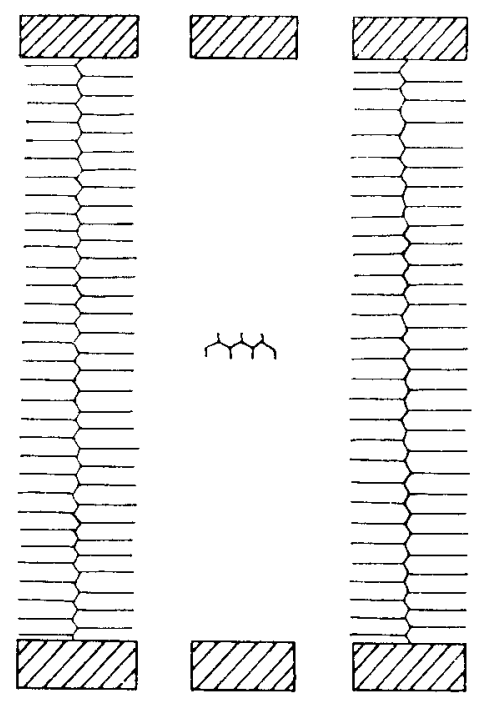

FIG. I. --.- Deux rayons de cire construite (vue supérieure) entourant une bande de cire gaufrée en position anormale.

\section{EXPÉRIENCES PRÉLIMINAIRES}

\section{Expérience $n^{\circ} 1$.}

Au sommet de la ruche est fixée sur une barrette de bois, entre deux cadres, un morceau de cire gaufrée de $20 \times 98 \mathrm{~mm}$; son plan est perpendiculaire aux rayons construits voisins.

En quelques minutes, des chaînes cirières visibles du haut de la ruche, s'établissent entre la languette de cire et les rayons voisins, tandis que s'effectuent les premiers étirages des cellules; ensuite, la feuille subit une torsion qui amène progressivement son sommet (partie opposée à la barrette de bois) dans une position parallèle au plan des rayons voisins. 
I a languette de cire prend ainsi la forme d'une hélice (fig. 2 et 3). Iin outre, les premières rangées des cellules de sa base (partie adjacente à la barrette) se trouvent déformées dans le sens vertical (fig. 4). C'est l'indice

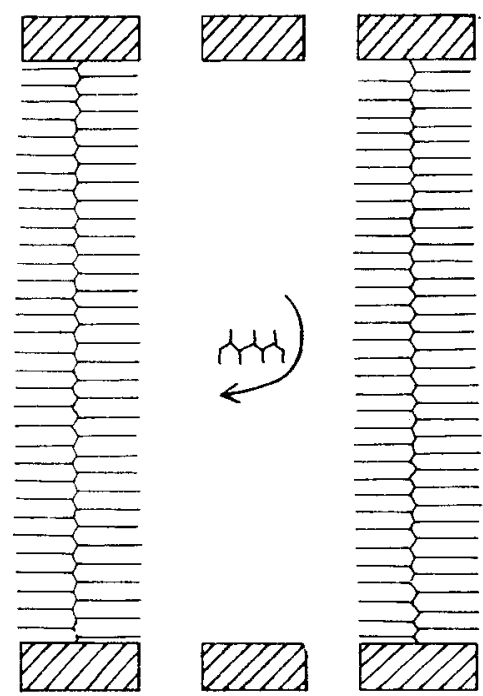

FıG. 2. - Résultat de l'expérience précédente : les Abeilles tordent la bande de cirt.

qu'un poids relativement important d'Abeilles s'y est suspendu. I a torsion elle-même ne pent s'expliquer que par des tractions musculaires des Abeilles accrochées les unes aux autres, d'une part au bord des lan-

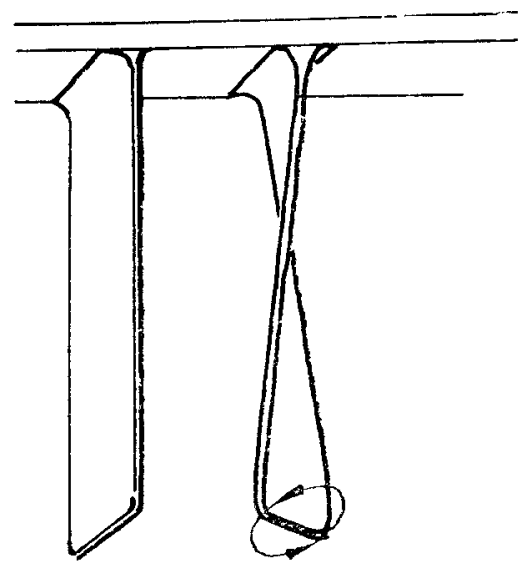

lite. 3. -.-Vue de protil : torsion de la cire gaufrée (à gatuche, le témoin ; à droite, le résultat).

guettes perpendiculaires, d'autre part, aux rayons construits les plus voisins. Or, une seule A beille est dans l'incapacité d'effectuer ce traiail: elle ne peut saisir à la fois le bord de la cire gaufrée et les rayons cons- 


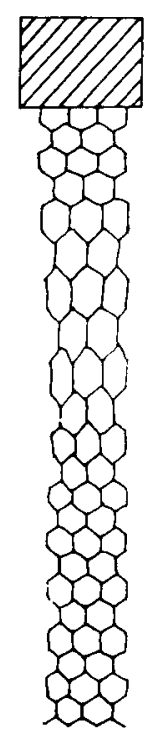

Frs. A. - sous l'ation du poids des Abeilles res chaines cirieres, les cellukes des languctes de cire gaufrée s'allongent verticalement.

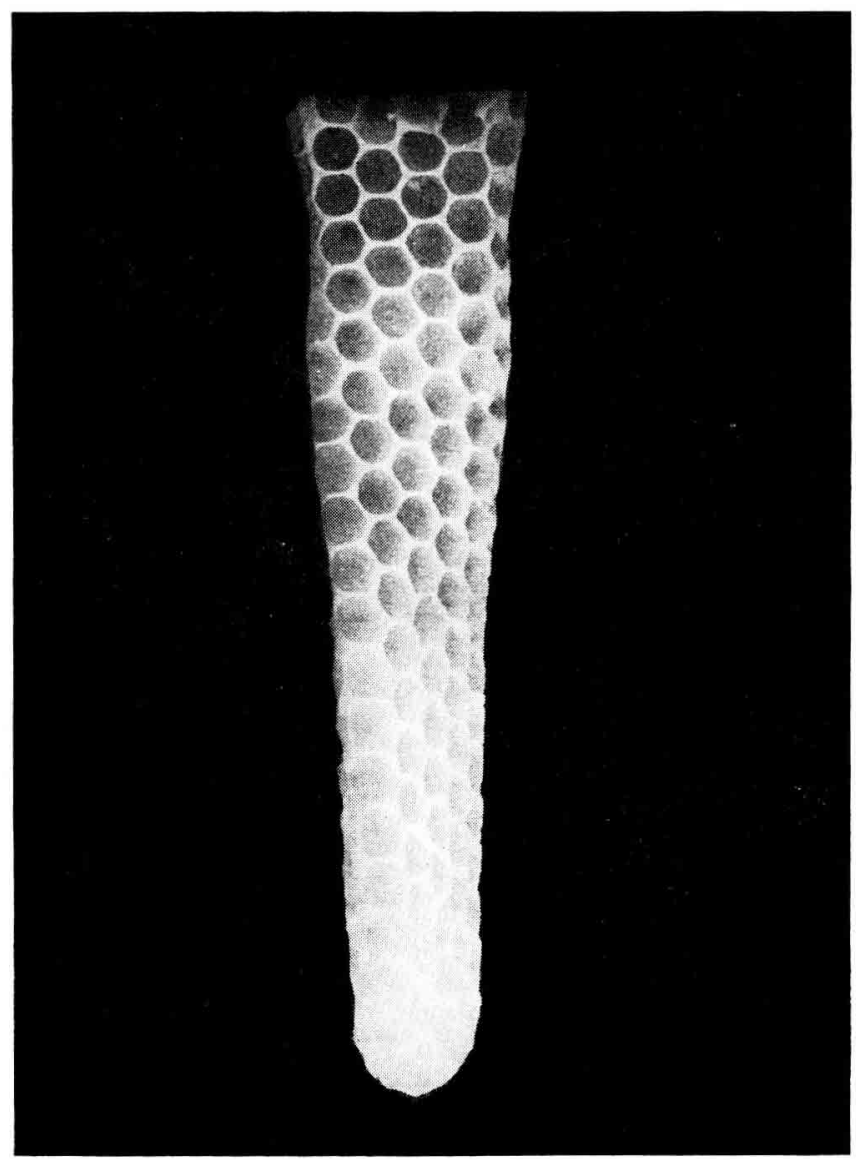

Photo I. 
truits; elle ne possède pas la force suffisante pour torctre la première et la rendre parallèle anx seconds. Nons allons: donc exanniner ce pliénonène qui implique obligatoirenent la collaboration d'un grand nombre d'inscetes occupés à la nême tâche.

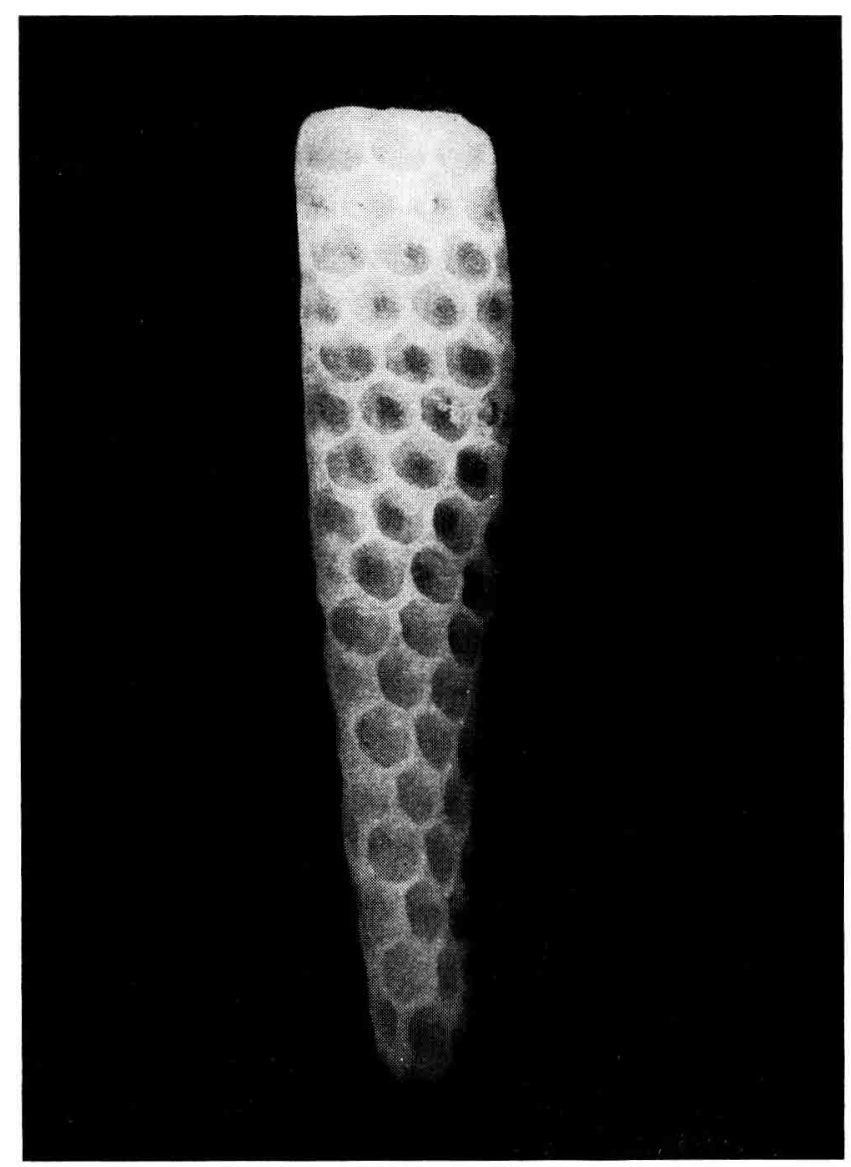

Photo2.

\section{Expérience $\mathrm{n}^{\circ} 2$.}

On peut tenter de dissocier le comportement d'étirage ou de construction de celui de torsion. Il suffit d'enduire la languette de cire d'une solution de propolis dans l'alcool, qui inhibe la construction (méthode de l'imladmi). Après quelques heures, on obtient alors la même torsion. sans la monindre élévation des bords des alvéoles et sans le moindre développenent latéral de la feuille. L'indépendance relative des denx comportements, d'étirage et de torsion, est donc mise en éridence. 


\section{Torsion et retomehes.}

Si l'on essaie de tordre un rayon déjà construit, on constate que cela est inpossible sans disloquer les parois des cellules qui se fondent en de nombrenx endroits. Or, lorsqu'on introduit perpendiculairenent à deux autres rayons un élément de rayon déjà construit, les Abeilles le tordent aisément, sans que les parois portent trace de rupture. (on voit, par contre, de nombreuses cellules défornées dont les parois rerticales forment des dièdres trop ouverts, la cellule s'aplatissant dans le sens horizontal. Ainsi donc les A beilles doivent remédier par des retonches successires aux inéritables lésions des parois an cours de la torsion.

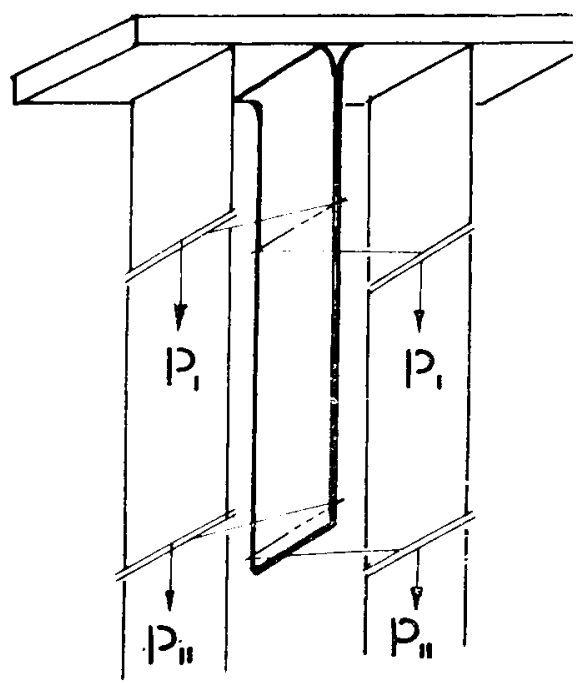

FIG. 5. - Constitution d'un modele mécanique pour torelre artificiellembent une languetue de cire ${ }^{3}{ }_{1}, \mathrm{I}_{11}$ représentent des forces.

\section{Essai de ronstitution d'un modìle.}

Avec C. ct M. Huris, nous avons essayé de tordre nons-mènes des lanlelles de cire gaulrée à une température légèrenent inféricure à celle du couvain de la ruche. Dans ces conditions, la cire est très molle et très malléable.

Sur we potence, nous fixons une bande de cire de $24 \times$ I fo $111 n$. Fin différents points de sa longueur à partir de son point de fixation et à son extrémité libre sont attachés, sur de petites tiges de métal traversant 1a bande de cire gaufréc de part en part, des fils très fins sur lesquels doivent s'appliquer des forces de traction. Ces tractions sont exercées par des masses de métal qui coulissent verticalement autour d'un axe horizontal (fig. 5). 
Dans ces conditions, il n'existe que deux façonss d'obtenir une torsion : 1a première $(a)$ consiste à appliquer une seule force de traction sur un seul bord à condition qu'un poids suffisant, à l'extrémité libre de la lamelle, maintienne la parfaite verticalité du système. Fn revanche, la deuxième $(b)$ demande l'application de deux tractions égales et en sens opposé, dans un plan horizontal sur chacun des deux bords verticaux de la lamelle.

Quel est le mode de traction utilisé par les insectes, sachant que les cellules proches de la barre de soutien semblent déformées par une traction verticale et qu'à la fin des expériences la lamelle de cire prend tune forme hélicoïdale?

A priori, il semble que les Abeilles utilisent la méthode $(a)$. Iin fait, cela devient douteux après deux séries d'expériences effectuées hors de la ruche à $29^{\circ}$ et à $32^{\circ}$; un poids de 2 g., fixé à l'extrémité de la feuille de cire,
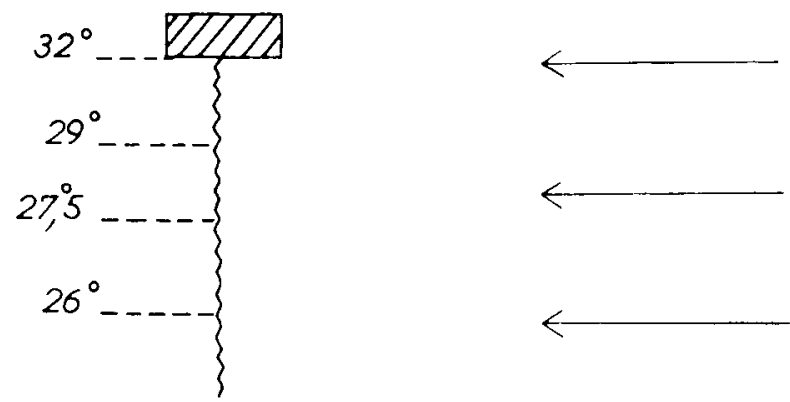

Fic. 6. - Répartition de la rhaleur sur une langruette de cire exposéc aux rayons infra-ronges.

est nécessaire et suffisant pour obtenir une déformation des cellules du haut sans aller jusqu'à la rupture. Or, à cette température, une force horizontale de $2 \mathrm{~g}$, insuffisante pour amener une torsion de $90^{\circ}$, déchire la cire. A $29^{\circ}$, les résultats sont semblables avec une traction horizontale de $5 \mathrm{~g}$ et une verticale de 6 ou $7 \mathrm{~g}$.

Dans les deux séries, l'extrémité libre de la cire est soulevée par la force horizontale, les cellules s'allongent et se rompent près de la barre de bois, la courbe de torsion est totalement différente sur les deux cotés verticaux de la lamelle.

Or, l'observation des rayons extraits des ruches nous montre que: Io I es cellules sont allongées à quelques centimètres au-dessous du support; $2^{\circ}$ I es cires tordues sont verticales; $3^{\circ}$ I es courbures des deux côtés sont seniblables.

I. - Dans la ruche, sur la languette de cire, la zone d'élongation des cellules au-dessous des supports correspond, semble-t-il, à la présence d'un plus grand nombre d'Abeilles qui doivent ramollir la cire. 
Dans mes modèles, les cellules des bandes de cire sont plus allongées immédiatement au-dessous de la barre de soutien. Or la température se répartit ainsi tout au long des bandes : $32^{\circ}$ près de la barre, $29^{\circ}$ au premier quart au-dessous, $27,5^{\circ}$ à la moitié et $26^{\circ}$ à l'extrémité libre (fig. 6). Pour les expériences suivantes avec les modèles, j'ai donc interposé un écran blanc opaque de $2 \mathrm{~cm}$ entre la source de chaleur (1) et la cire gaufréc soudée à la barre transversale. Je relève alors une nouvelle répartition de la chaleur : $30,5^{\circ}$ près de la barre, $31,5^{\circ}$ légèrement au-dessous de 1'écran, $3^{0}, 5^{\circ}$ à la moitié (fig. 7).

res résultats furent exactement les mênes, hormis la déchirure qui s'effectua au point de température maximum à quelques centimètres de la barre transversale. En cet endroit, les cellules sont plus allongées.
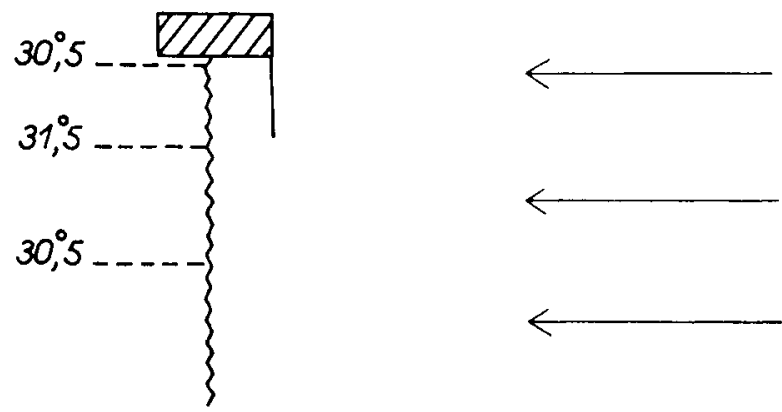

FIG. 7. - Modification de la répartition de la chaleur au moyen d'un écran sur une languette de cire exposée aux rayons infra-rouges.

II. - I)ans les ruches, les rayons tordus restent verticaux. I1 nous faut donc obtenir des modèles qui présentent la même particularité. Nous avons vu que cela ne se peut qu'avec une force verticale ne dépassant pas $2 \mathrm{~g}$ à $32^{\circ}$ et $5 \mathrm{~g}$ à $25^{\circ}$ et un couple de forces horizontales. Ceci laisse donc supposer que, dans la ruche, le poids des Abeilles suspendues anx languettes de cire ne dépasse pas $2 \mathrm{~g}$ à $32^{\circ}$.

III. - Enfin, on doit retrouver dans nos modèles des courbures de torsions semblables sur chaque arête de la languette. Seuls deux couples accrochés sur les côtés de la cire gaufrée et tirant horizontalement en sens opposé, nous ont permis d'atteindre ce but. I a cire restait alors verticalc, et ne se rompait pas.

Les forces qui nous permettent d'obtenir des modèles ressemblant le plus possible aux édifices des Abeilles, sont les suivantes : à $32^{\circ}$, une traction verticale de $2 \mathrm{~g}$; deux forces horizontales opposées égales de $2 \mathrm{~g}$ chacune; à $29^{\circ}$ une traction verticale de $5 \mathrm{~g}$ et 2 latérales de $6 \mathrm{~g}$.

(1) Une lampe à infrarouges horizontale, placéc à $\mathbf{~ c m}$ en face du modèle. 
Il ne reste plus qu'à rechercher les points d'application de ces forces. I.es meilleurs semblent être situés au-dessus de la première moitié du rayon : au-dessous de cet endroit les courbures sont trop faibles, au-dessus, elles sont trop fortes.

Il va sans dire que, dans la ruche, ces deux forces du couple expérimental ne représentent en fait que les résultantes d'un plus grand nonbre de tractions réparties tout au long des bords de la languette de cire.

Enfin, notons-le bien, il est commun de voir des Abeilles se tenant par les pattes et tirant sur les bords de la feuille de cire pour la redresser : deux Abeilles peuvent suffire pour relier un des bords de la bande de cire ot les cellules du plus éloigné des rayons voisins.

On peut donc conclure, semble-t-il, que l'étude du modèle de cire nous conduit à admettre que, dans la ruche, seule la présence de couples de triction égaux, horizontaux et de sens opposé explique la création de languette. de cire de forme hélicoïdale.

\section{POINTS D'APPLICATION DES CHAINES CIRIÈRES}

Des écrans de toutes tailles et de toutes formes placés à différents endroits à proximité des bandes de cire donnent des indications supplémentaires.

I. - Ie larges feuilles de métal, placées sur les deux rayons voisins const ruits empêchent le développement et la torsion de la cire placée entre elles. D'ailleurs, on peut diminuer progressivement la largeur de l'écran jusqu'à ro mm sans pour autant permettre une torsion. Mais par contre, il faut absolument maintenir ces écrans bien en face des arêtes du rayon perpendiculaire si l'on ne veut pas la voir apparaitre. Ces résultats confirment donc mes conclusions sur l'existence de zones sensibles (les tranches) dans un rayon en construction.

2. - Fin revanche, la moindre ouverture (supérieure à ro mm de large) verticale, percée dans toute la longueur dans un seul des écrans de 30 à 2 ro mm de large, suffit pour faire réapparaître la torsion : d'ailleurs, cette fente peut être remplacée par de petites fenêtres carrées de $16 \mathrm{1mm}$ de côté. Rappelons encore les résultats de travaux antérieurs (DARCHis, I956) : un petit orifice à 1'intérieur d'un écran placé en face d'une languette (le cire sur un rayon parallèle extrêmement rapproché déterminait une courbure qui tendait à la ramener dans une position plus éloignée. Cette régulation n'existait pas en l'absence de l'orifice.

3. - Mais, on le sait, les Abeilles couvrent d'un opercule les cellules contenant du couvain et du miel. Cependant, ces opercules sont bien différents : ceux du couvain légèrenlent bombés et réguliers, épousent la forme hexagonale de la cellule, ceux du miel sont plats et informes. Voilà peut-être la raison pour laquelle les cirières déploient un compor- 
tement totalement différent lorsqu'elles trouvent en face d'une bande de cire gaufrée, fixée comme dans les expériences précédentes, deux rayons voisins composés de cellules de miel ou de convain : en face $d u$ contain, elles opèrent la régulation, en face du miel elles étivent la bande et la collent aux opercules mais sans torsion. Ce phénomène rappelle fortement celui que nous avons signalé il y a quelques années : les Abeilles écartent un rayon anormalement rapproché d'un autre, si elles découvrent en face de lui de la cire formée en cellules (DARCHEN, I954).

\section{SENS ET AMPLITUDE DES TORSIONS}

I. - Pour déterminer le sens de la torsion d'une bande de cire nous la retournons et l'observons par son extrémité libre; le sens alors est dextre, sens des aiguilles d'une montre, ou sénestre.

Tous avons effectué 49 expériences : 22 donnent des torsions dans le sens sénestre, 27 dans le sens dextre. On peut dive que la torsion se fait an hasard.

Cependant, si l'on analyse la répartition des résultats ruche par ruche, on s'aperçoit que certaines d'entre elles présentent des tendances à tordre les rayons dans un sens ou dans un autre.

\section{TABLEAL I}

\begin{tabular}{c|c|c}
\hline Torsions & Senestres & Dextres \\
\hline A & 7 & 3 \\
I & 7 & 16 \\
C & 3 & 7 \\
D & 2 & I \\
I & 3 & 0 \\
\hline Total ............ & 22 & 27 \\
\hline
\end{tabular}

Notons que les expériences dans une même ruche sont à peu près toutes effectuées au même endroit et que les stimuli issus de l'environnement doivent être à peu près les mêmes pendant toutes les expériences. Pourtant, pour connaître l'action des rayons voisins sur le sens des torsions, dans la ruche (A), j'ai remplacé une fois l'un par l'autre les cadres construits à gauche et à droite de la languette de cire, mais de telle sorte que les mêmes faces des rayons restent tournées vers la bande de cire : la tendance à tordre les rayons dans le sens précédent s'est maintenue pendant toute la durée de l'expérience, c'est-à-dire pendant plusieurs jours.

Remarquons enfin que, si l'on fixe aux extrémités d'une même barre de bois deux bandes de cire gaufrée, les sens de torsion sont souvent les mêmes ( 13 fois sur 20 environ). 
2. - Lin maintenant le matériel expérinental au mêne endroit, il est possible d'intercaler un écran de $200 \mathrm{~mm}$ de large entre la languette de cire et l'un des rayous voisins : en regardant la ruche par derrière, l'écran peut être à gauche, il y a alors,; torsions sénestres ponr 7 dextres ; s'il est à droite, les torsions sénestres sont au nombre de ro et les dextres au nombre de 4 . Il semble done que la position de l'écran joue sur le sens de la torsion.

'TaBifial II. Amplinde des torsions de la cive ganfrée.

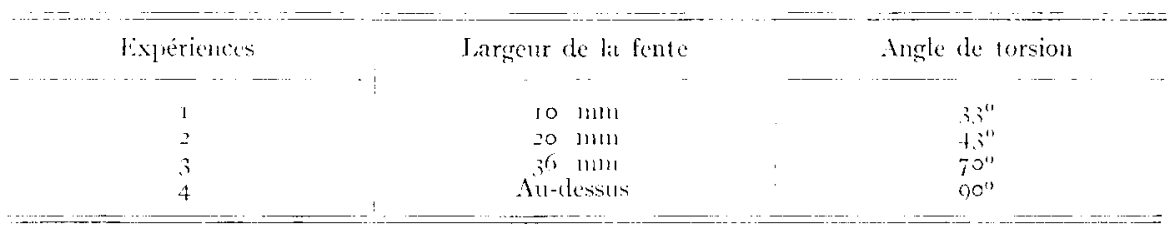



Flg. 8. -..- Deux petits rayons construits et perpendiculaires entre eux sont soudés l'un à l'autre entre deux cadres à l'intéricur d'une ruche : la partic (A) est parallele aux rayons voisins, la partie (B) est perpendiculaire.

Cependant, il faudrait recommencer less mêmes expériences sur d'autres ruches, car nous savons que certaines d'entre elles peuvent présenter des tendances à donner aux torsions des sens bien déterminés. '́outefois, nos expériences possèdent une valeur assez significative puisqu'elles ont été effectuées sur les ruches "dextres".

3. - Nous avons déjà signalé qu'une fente verticale dans l'écran métallique face à la lamelle de cire suffit à y induire une torsion même si le cadre voisin et opposé est recouvert d'un écran de métal. Toutefois, l'amplitude de la torsion semble proportionnelle à la largeur de l'ouverture; le tableatu II ci-dessus nous le montre suffisamment. 
J'ai répété les mênnes expériences en remplaçant la languette de cire gaufrée par denx petits morceaux de cire construite formant tne croix : 1e premier est parallèle aux rayons voisins et soudé à la barrette de bois qui sert de support, le second est fixé perpendiculairement au sonmet du précédent (fig. 8).

I'amplitude de la torsion est rapportée dans le tablean (III). I,es résultats de ces expériences sont comparables aux précédents. Toutefois, les tbeilles semblent exiger alors une ouverture plus grande de l'écran pour tordre complètement less rayonss de cire construite.

TABLIFAU III

Amplitude des torsions d'un rayon construit.

\begin{tabular}{c|c|c} 
Fxpériences & Largeur de la fente & Angle de 1orsion \\
\hline $\mathrm{I}$ & $10 \mathrm{~mm}$ & $3^{8^{\circ}}$ \\
2 & $14 \mathrm{~mm}$ & $45^{\circ}$ \\
3 & $74 \mathrm{~mm}$ & $70^{\circ}$ \\
4 & Au-dessus & $90^{\circ}$ \\
\hline
\end{tabular}

Mais, rapidement, le long du rayon fixé à la barre de soutien, parallèles aux rayons construits, se développent des protubérances verticales, lorsque la fente est inférieure ou égale à $\mathrm{I}_{4} \mathrm{~mm}$.

\section{QUELQUES PROBLEMES DIFFICILES A RÉSOUDRE POUR LES ABEILLES}

\section{Expérience $\mathbf{n}^{\circ} 1$.}

La bande de cire gaufrée est fixée à l'intérieur de la ruche, à la barre inférieure d'un calre, au milieu d'une forte population d'Abeilles. Comme elle a tendance à s'affaisser, elle est maintenue par un fil à coudre dans un plan vertical orthogonal au plan du cadre (fig. 9). Au bout de denx heures, les résnltats sont les suivants : 1a bande subit une rotation très faible. Tous employons le terme de rotation car la bande reste plane et c'est ce plan tout entier qui fait $11 n$ angle assez faible avec la position primitive du plan de cette bande : il n'y a pas de torsion hélicoüdale comme celle obtentue lors des expériences précédentes. Ces expériences 11'ont été réalisées que deux fois.

\section{Expérience $\mathrm{n}^{\circ} 2$.}

Puisque les Abeilles tordent ainsi les feuilles de cire gaufrée pour les replacer dans un plan parallèle à celui des rayons voisins, il vient immédiatement à l'esprit de fixer bout à bout deux morceaux de cire gaufrée 
perpendiculaires : l'un, adjacent à la barre de bois, se tronve dans un plan orthogonal à celui des rayons voisins (partie A), l'autre dans un plan parallèle (partie B) (fig. Io). I es deux mesurent 4 cellules de large et

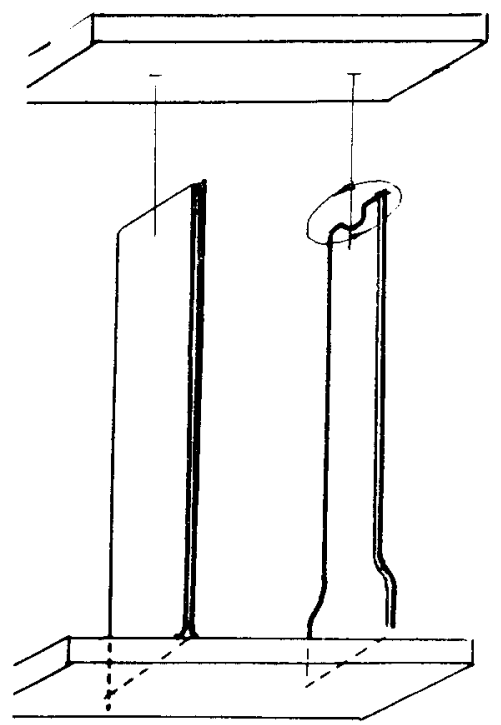

FIG. 9. - Une cire gaufrée est fixée à l'intérieur de la ruche, ì la barre inférieure du cadre. Un fil soutient l'ensemble : à gauche, présentation de l'expérience, à droite, résultiıts.
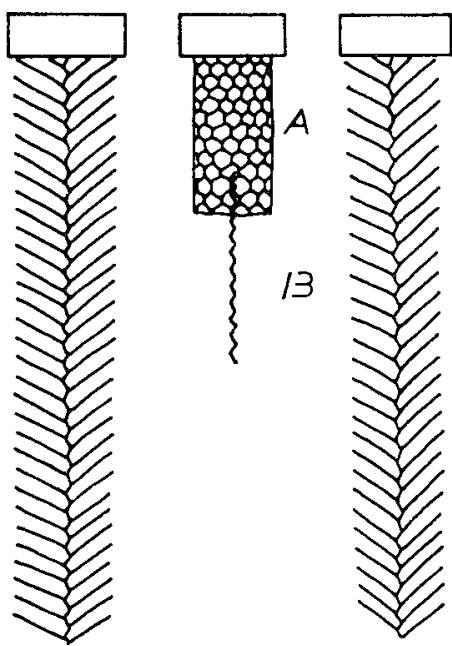

Fíg. Io. - Deux languettes de cire attachées l'une à l'autre sont soudées sur une barrette de bois entre deux rayons à l'intérieur d'une ruche.

$I_{5}$ de long et sont encastrés l'un dans l'autre sur une longueur de 5 cellules. Quelle va être la solution adoptée par les Abeilles puisqu'elles se trouvent en face d'un problème complexe fondé sur le conflit entre deux 
tendances : tordre la partie du rayon qui est perpendiculaire revient à mettre en position anormale la partie inférieure qui était parallèle anx faces?

Au premier stade de la régulation, dans les heures qui suivent le début de l'expérience, les Abeilles tirent sur la feuille de cire orthogonale (A) et lui font exécuter une torsion de $23^{\circ}$. Mais au même moment, la languette de cire inférieure $(B)$, parallèle anx rayons voisins, subit la même torsion relative et prend ainsi une disposition anormale. A la fin de ce premier stade, l'amplitude de la torsion n'augmentera plus. An deuxième stade, les morceaux de cire restent dans leurs positions respectives, nais par des étirages et des constructions latérales, les Abeilles

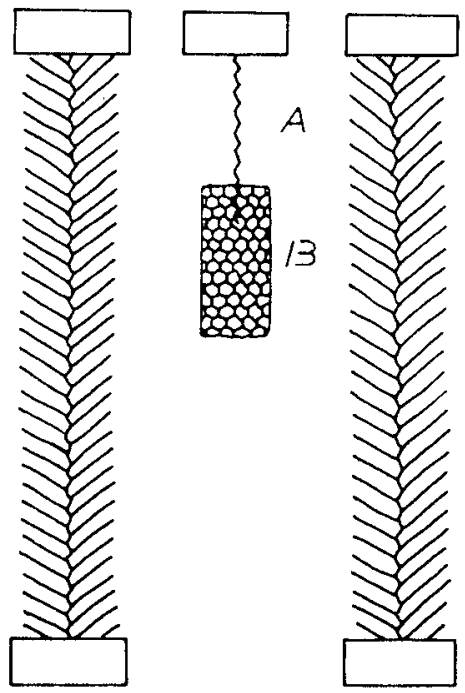

Fig. 11.-.- Deux bandes de ciregaufrée soudines bout à bout sont interalées entre deux rayons construits.

ramènent le rayon inférieur et parallèle aux faces des rayons voisins au début de l'expérience (B), à $5^{\circ}$ de sa position primitive. lin revanche, le rayon adjacent à la barre de bois (A) croît dans le sens qui lui a été donné à la fin du premier stade, c'est-à-dire dans un plan situé à $23^{\circ}$ par rapport au plan orthogonal primitif. İnfin, des côtés du premier rayon adjacent à la barre transversale (A) naissent des protubérances verticales de cire qui se développent le long du second rayon (B) et se soudent à lui.

\section{Expérience $\mathbf{n}^{\circ} 3$.}

Je n'ai pas effectué l'expérience qui consiste à changer les positions des languettes de cire : languette en position normale près de la barre, languette orthogonale au-dessous (fig. II). Cependant, je crois qu'il est 
utile de rapporter ici les réstultats de nombrenses expériences effectuées avec de petits rayons construits placés dans less positions suivantes (fig. s): rayon parallèle anx voisins, adjacent à la barre de bois (A), rayon orthogonal an sommet du précédent (B). Ce dernier est alors toriu, démoli en partie et reconstruit : il prend une forme hélicoïdale dont le sonnet est parallèle aux rayons voisins. Simnltanément les nonveanx éditices du rayon anormalement placé près de la barre transversale (A) ne sont plus construits dans le plan primitif, parallèle anx rayons voisins, car les fonds des nonvelles cellules se situent dans un nonvean plan qui tente d'unir les deux rayons construits. Iinfin, l'ensemble des constructions est sans cesse retottché.

Io Bref, quelle que soit donc la position du rayon anomalement placé, il est toujours redressé au moins en partie. 20 I a torsion se répercute toujours plus ou moins profondément sur le rayon fixé parallèlement aux rayons voisins. $3^{\circ}$ Dans l'expérience 2 , 1'amplitude le la torsion semble être la résultante de l'action de forces qui tentent à redresser la languette anormale et à garder dans sa position la languette normale: il y a peut-être une traction inverse des channes fixées sur les deux morceaux de cire. $4^{0}$ I'intercorrélation continuelle des grappes cirièress à tous les niveaux de l'édifice paraît démontrée. $5^{\circ}$ I 'achèvement de la régulation (torsion totale) se fait par des constructions latérales nouvelles et par des remanienents de la cire construite.

\section{CONCLUSION}

Ia torsion des cires gaufrées constitue un exemple de plus des régnlations si précises et si complexes observées dans les constructions de divers insectes sociaux comme les Termites ((irassí), les Fourmis oecophylles (LEDoux, Cindurin) et dans d'autres expériences chez les Abeilles elles-mêmes (DARchis, en cours de publication).

I es descriptions du travail des (licophylles nous montrent que ces insectes forment aussi des chaînes chargées de rapprocher les bords d'une ou de plusieurs feuilles de Caféier : les ouvrières de ces chaînes sont parallèles et conjuguent leurs efforts en tirant dans le même sens. A ce sujet, I,EDOC pose la question suivante: $Y$-a-t-il coordination dans le travail collectif des onvrières ou simple action côte à cote sans souci de la voisine? Il conclut que, si les (Ficophylles possèdent un " réflexe simple de traction ", on ne doit pas oublier que tous ces réflexes sont simultanés et tendent au même moment à la réalisation du mêne but dans des situations différentes. Cun vax insiste au contraire sur les techniques variées ntilisées par ces fourmis pour arriver à former des nids dans des circonstances et avec 111 matériel foliaire extrênement divers. Ce dernier auteur admet donc que l'(ricophylle, tordant wne feuille quelconfue, 
répond à un ensemble de stimuli plus ou moins nombreux mais non pas simples : 1a réponse de construction de la fourmi dépend d'une intégration de nombreux facteurs issus de l'environnement, d'un " complexe de stimulations".

La réponse est encore plus compliquée chez l'Abeille si l'on admet l'existence des couples de traction qui réalisent quelquefois une torsion de $90^{\circ}$ dans les deux premières heures qui stivent la pose de l'expérience. Chaque Abeille agit-elle indépendamment sans se préoccuper de sa voisine? Alors pourquoi tire-t-elle dans un sens plutôt que dans un autre puisque apparemment les deux solutions sont possibles? Pourquoi s'attache-t-elle au bord de la cire gaufrée, au bord le plus éloigné le seul qui puisse amener la torsion, mais aussi celıi qui exige la présence d'une chaîne cirière de traction?

Pourquoi, en général, le poids des Abeilles, suspendues à la lamelle de cire, n'excède-t-il pas celui qui amènerait la rupture de la cire?

Chez l'Abeille du moins, on ne peut admettre que la présence de réflexes simples soit à l'origine de la régulation décrite ici. Pour autant, parlerons-nous d'une compréhension (insight) du problème par les Abeilles qui doivent le résoudre? Mais n'y-a-t-il vraiment que cette alternative? Nous préférons attendre de plus amples informations, fondées sur l'observation des constructions à travers des ruches vitrées.

\section{RÉSUMÉ}

Io Nous montrons que les chaînes d'Abeilles jouent un rôle important dans les régulations concernant le parallèlisme des rayons. En particulier, elles peuvent faire subir une torsion de $90^{\circ}$ à une lamelle de cire gaufrée fixée en haut d'une ruche dans un plan orthogonal aux plans des rayons voisins.

$2^{\circ}$ La construction d'un modèle mécanique nous démontre que seules des tractions horizontales (couples de forces opposées) sontsu sceptibles de rendre compte de la régulation décrite.

$3^{0}$ Les points d'application de ces chaînes régulatrices se trouvent sur les rayons construits.

$4^{\circ}$ Il $11^{\prime} y$ a pas de sens privilégié de torsion mais des tendances propres à chaque ruche.

$5^{\circ}$ Un jeu d'écrans solides de différentes formes et tailles, disposés à divers endroits des rayons voisins nous indique que les torsions s'effectuent toujours lorsqu'une faible partie d'un des rayons voisins est découverte en face de la tranche de la lamelle de cire. A cette occasion, nous rappelons les résultats similaires que nous avons obtenus il y a quelques années à propos de courbures imprimées aux rayons situés trop près de leurs voisins. 
$6^{\circ}$ Nous indiquons les moyens de faire varier l'amplitude et le sens de torsion.

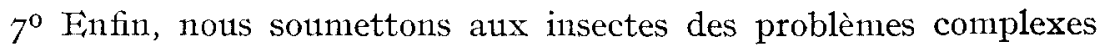
dans lesquels entrent en conflit les tendances à tordre les rayons et à en garder d'autres dans leurs positions initiales.

\section{REFFERENCES BIBLIOGRAPHIQUES}

Chauvix (R.). - Sur la reconstruction du nid che\% les fourmis (Ecophylles. Behaviour, 4, I90-20I, I952.

Darchen (R.). - Quelques régulations sociales chez les Abeilles. Ins. Soc., $1,219-228$, I954.

Les constructions sociales chez A pis mellifica. Proc. Ioth Intern. Congr. Entom., $2,529538, \quad 1956$ (1958).

Grasse (P. P.). - Ia reconstruction du nid et le travail collectif chez le Termite supérieur. J. Psychol., 370-376, I939.

LEDoux (A.). - Étude du comportement et de la biologie de la fourmi fileuse des tropiques. Thèse de Doct. 157 p., Paris, I949.

I.FDoux (A.). - Recherches sur la biologie de la fourmi fileuse Ecophylla longinoda Latr. An. Sc. Nat. Zool., 12, 314-46I, I950. 